\title{
EL PROYECTO HIDROELÉCTRICO DE JALCOMULCO, VERACRUZ, MÉXICO, Y LAS REACCIONES DE LA SOCIEDAD CIVIL
}

\author{
Nelly Josefa León Fuentes*
}

\begin{abstract}
Resumen: Este artículo aborda una de las problemáticas que enfrenta el estado de Veracruz en los últimos cuatro años en relación con las concesiones otorgadas a empresas internacionales para la realización de varios proyectos hidroeléctricos. Toma como ejemplo el caso del proyecto multipropósitos Xalapa, en el río los Pescados en Jalcomulco, ubicado cerca de la capital del estado de Veracruz, para analizar las reacciones de la población afectada y el tipo de conflicto que se ha generado, considerando las capacidades económicas, sociales y culturales de la población que padece el proceso de invasión territorial y de sus recursos, y la acción de las organizaciones civiles. El análisis está apoyado en la historia ambiental con el uso de la historia oral como estrategia, puesto que la resistencia continúa vigente y los testimonios de los actores protagónicos son fundamentales para la comprensión integral de los procesos de apropiación y despojo, con lo cual se da voz a los grupos excluidos.
\end{abstract}

Palabras clave: Represas hidroeléctricas, movimientos sociales, organizaciones civiles, conflicto ambiental, Veracruz, México.

\begin{abstract}
This article cover one of the problems what confront the state of Veracruz over the last four years: it concerns the concessions granted to international companies for the development of various hydroelectric projects. In particular, the study takes up the case of the multi-purpose project Xalapa, situated on the Pescados River, in Jalcomulco, near the Veracruz state capital. This allows us to analyse the reactions of the inhabitants affected, and the type of conflict generated, taking into consideration their economic, social, and cultural capabilities, the resources at their disposal, and the actions of civil organisations. Analysis rests on environmental history, employing oral history as a strategy given that resistence is ongoing and actors' testimonies are fundamental for an overall understanding of the processes of apropriation and dispossession: this route allows the 'excluded' to be heard.
\end{abstract}

Fecha de recepción: 23/09/2015 - Fecha de aceptación: 20/11/2015

* Mexicana. Doctora en Historia y Estudios Regionales, Universidad Veracruzana (UV), México. Docente en la Facultad de Sociología del Sistema de Enseñanza Abierta e integrante del CA: Ciencias Sociales e Interdisciplinariedad, UV. Correo electrónico: fuentesnj82@gmail.com 
Keywords: hydroelectric dams, social movements, civil organizations, environmental conflict, Veracruz, México.

"Yo hablo por el río. No a las presas".
Grupo Centinela del río Los Pescados

\section{Introducción}

$\longrightarrow$

1 presente trabajo tiene por objetivo abordar la problemática que se ha presentado en los últimos años en el estado de Veracruz, desde que el gobierno de Fidel Herrera (2004-2010) otorgó concesiones a empresas internacionales para que en el territorio veracruzano se realizaran varios proyectos hidroeléctricos, sin tomar en cuenta ni a sus habitantes ni la afectación de sus territorios. De ahí que el tema sirva para observar cuáles han sido las reacciones de la población afectada y qué tipo de conflicto se ha generado en cuanto a las capacidades económicas, sociales y culturales de la población que padece el proceso de invasión territorial y de sus recursos, así como de los apoyos externos que recibe de las organizaciones civiles. Para abordar esta problemática se tomó como estudio de caso el proyecto de construcción de la presa hidroeléctrica con inversiones de la empresa brasileña Odebrecht y del gobierno estatal en la barranca del río los Pescados en Jalcomulco, Veracruz. Este lugar se ubica 41,5 $\mathrm{km}$, de Xalapa, capital del estado, por tanto, eje de los poderes de gobierno y núcleo educativo importante por una carretera interna pavimentada que va hacia Orizaba, atravesando el municipio de Coatepec por la zona de Tuzamapan, en el centro del estado de Veracruz -véase mapa 1-.

Es importante destacar que el interés del tema surgió porque desde hace tiempo se ha proyectado la construcción de aproximadamente 112 presas de diverso tamaño -la mayoría consideradas mini hidroeléctricas- en el estado de Veracruz, en México. De las 300 presas programadas en la franja entre México y Panamá dentro del Programa de Integración Energético Mesoamericano (PIEM) firmado en 2006, en el que participan Belice, Colombia, Costa Rica, El Salvador, Guatemala, Honduras, México, Nicaragua y Panamá como países miembros, donde diversas empresas de capital internacional aprovechan la apertura comercial promovida por la fase neoliberal para beneficiarse de los potenciales hidráulicos de toda esta zona. En el caso de Méxicouna, sola entidad mexicana -Veracruz-, con sus fluentes de agua aportará 37\% de la energía para este programa, a pesar de que los ríos más caudalosos ya han sido aprovechados en la generación de energía.

El plan Puebla Panamá tiene la intención oficial de favorecer la competitividad económica de la región centroamericana a través del otorgamiento de permisos y concesiones para que el capital privado pueda desarrollar vías de 


\section{Mapa 1 \\ Ubicación de la región afectada de Jalcomulco}

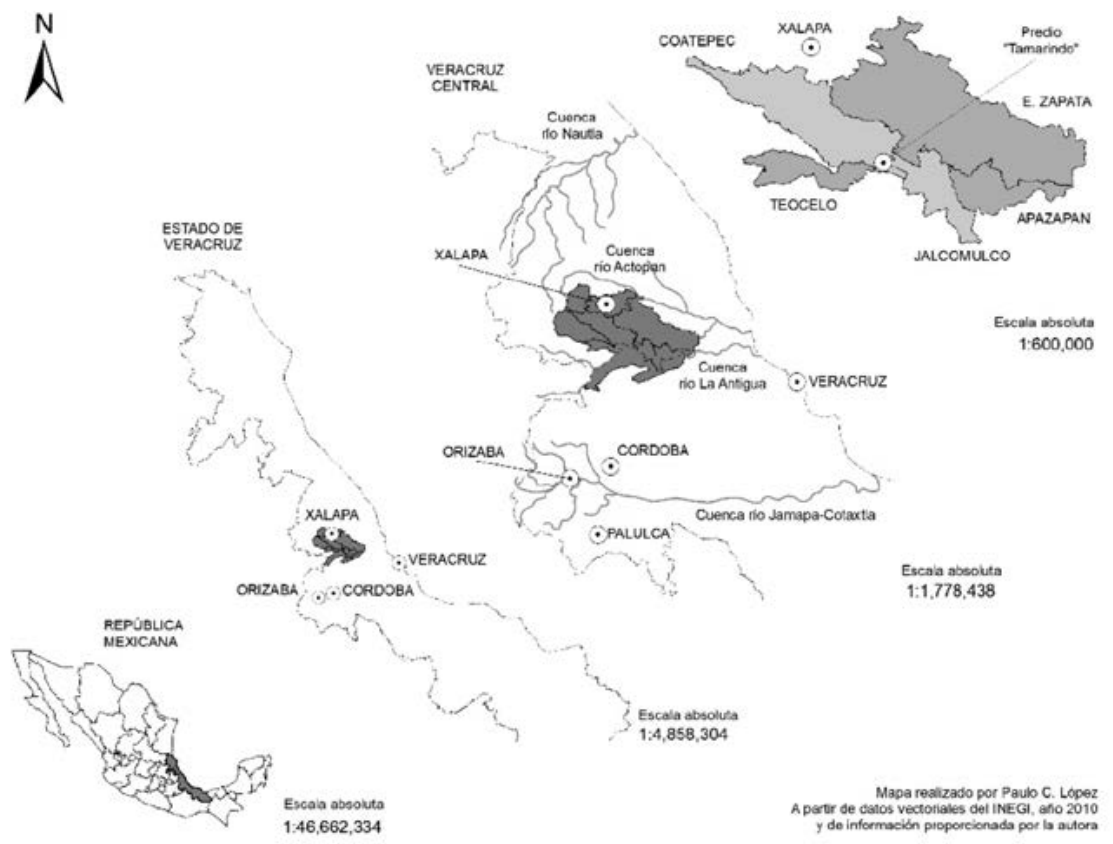

Fuente: Elaboración a partir de datos del Instituto Nacional de Estadística y Geografía (INEGI), México, 2010.

comunicación e hidroeléctricas que permitan el auge industrial, tecnológico, comercial y turístico que acabe con los problemas de salud, las limitaciones al comercio y la pobreza de la zona; aunque el trasfondo sea proveer un escenario propicio para las inversiones del capital privado; pues en particular, respecto a una parte del programa, busca generar y acumular energía eléctrica en grandes cantidades para su exportación a través del Sistema de Interconexión Energética de América Central (SIEPAC) y la red mexicana de interconexión eléctrica. ${ }^{1}$ Parte del interés de los entes participantes es que el agua y la energía sean para surtir a las poblaciones urbanas y a las diversas ramas de la economía. La instalación de dichas presas frecuentemente ha generado oposición y resistencia de parte de las poblaciones afectadas, cuyas acciones de protesta son bastante distintas según sus condiciones sociales, económicas y culturales, en algunos casos se

1 Rodolfo Chávez Galindo, "El conflicto de la presa la Parota", en: Estado del desarrollo económico y social de los pueblos indígenas del gobierno de Guerrero (México: Universidad Nacional Autónoma de México - UNAM/Programa Universitario; México Nación Multicultural/Secretaría de Asuntos Indígenas del Gobierno del Estado de Guerrero, 2009) y Karla Alethya Jara Duran, Balance de la situación de las presas en México en el siglo XX (Tesis de Licenciatura en Economía, Universidad Nacional Autónoma de México - UNAM, 2009). 
invaden áreas naturales protegidas o reservas indígenas; ${ }^{2}$ en otros, amplias áreas de cultivo para el mercado, así como espacios turísticos. Por esta razón se seleccionó un estudio de caso específico, donde se puedan observar las reacciones de la sociedad civil frente a un proyecto hidroeléctrico que altera las actividades económicas y productivas para los diversos actores sociales de la zona implicada.

La investigación forma parte del proyecto El agua en la Historia de Xalapa. Los retos del agua desde la épocacolonial al siglo $X X,{ }^{3}$ aunque la génesis del tema fue motivada por la información recurrente en la prensa para tomarla como "pretexto" en la enseñanza del curso taller de metodología de investigación con un enfoque cualitativo. De ahí que, el presente estudio esté basado en observaciones sistemáticas y entrevistas que apoyan la construcción de la historia oral, complementada con la información del plan de desarrollo municipal -hecho por sujetos ciudadanos, personas expertas y autoridades ejidales y municipales- prensa escrita y virtual, ya que es importante la cobertura mediática, pues el papel de los medios evita o vuelve más costosas las reacciones represivas del Estado en contra de quienes buscan solucionar un conflicto. En este sentido, los medios de comunicación ${ }^{4}$ desempeñan un rol determinante en los diversos ámbitos de la sociedad mexicana.

El propósito es explicar cómo el sistema capitalista en su fase neoliberal actual representa una forma de mayor depredación por las acciones coercitivas que se ejercen en las demarcaciones territoriales que ocupa para su construcción y desarrollo, lo que ha provocado una reacción de resistencia generalizada. Esta situación no es nueva, como señala David Harvey, ${ }^{5}$ pero hoy tiene mayores repercusiones por la situación económica que se pervive, cuando el reparto del mundo realizado por las trasnacionales es un hecho incuestionable, que continúa ejerciendo un dominio casi permanente y se expresa en la operación de diversos proyectos hidroeléctricos y mega-proyectos mineros, que para su puesta en marcha requieren de un gran almacenamiento de agua que implica el desvío

2 Por mencionar un ejemplo, véase el caso del mega proyecto hidroeléctrico "El Diquís" en Costa Rica, que ha afectado a las comunidades indígenas Térraba y aunque se apoye en grupos activistas para frenar el proyecto, debido a que comenzó sin previa información y consulta de los pueblos indígenas, violando así la legislación costarricense y el convenio 169 de la OIT firmado por Costa Rica desde 1993, el cual sugiere que para realizar obras en territorios indígenas es obligatoria la consulta a la población implicada, como esto no se ha hecho, la obra se detuvo. Instituto Costarricense de Electricidad (ICE), Una mirada al proyecto hidroeléctrico El Diquís, Costa Rica (Puntarenas, Costa Rica, ICE, s. f.), disponible en: http://pheldiquis. cr/Portals/0/Impresos/FolletoPHED.pdf. "Detrás Del Diquis. La verdad sobre el proyecto hidroeléctrico", video de Contra el PH Diquis, 1 de agosto de 2010 https://www.youtube.com/watch? $=x 1$ uuIYNaOls

3 Proyecto inscrito en el Sistema de Registro y Evaluación de la Investigación de la Universidad Veracruzana, además de ser parte de las actividades del CA Ciencias sociales e interdisciplinariedad y la Línea de Generación: Región, medio ambiente y sociedad.

4 Algunos de los medios que han apoyado el movimiento son: La Jornada, revista Proceso, ABC noticias, La Jornada Veracruz, Diario de Xalapa, diario La Política desde Veracruz y Al Calor Político. http:// www.imagendelgolfo.com.mx/resumen.php?id=41007695

5 David Harvey, El nuevo imperialismo. Acumulación por desposesión (Buenos Aires, Argentina: Consejo Latinoamericano de Ciencias Sociales - CLACSO, 2005). 
de los ríos, arroyos y manantiales, por lo que se hace necesaria la construcción de infraestructura que concentre corrientes de agua. Esta infraestructura daña social, cultural y ambientalmente a poblaciones y territorios de comunidades étnicas o campesinas, y hace que la reproducción social sea menos sustentable.

Como se trata de un movimiento reciente, para lograr objetivar de mejor modo este trabajo, la metodología se apoya en la perspectiva de la historia ambiental y en el enfoque de la historia inmediata, ${ }^{6}$ porque se abordan temas del presente, observando los hechos humanos del pasado y usando como estrategia la historia oral -la memoria viva- que se argumenta con los testimonios obtenidos a través entrevistas con personas que han protagonizado el movimiento para tener un conocimiento directo, a fin de construir el relato histórico, dando voz a los excluidos y buscando la complementación con datos sobre el hecho histórico-social para que la subjetividad sea menos. Sobre todo, para entender los procesos sociales y comprender las dinámicas ambientales, económicas y culturales del territorio veracruzano. ${ }^{7}$

La hipótesis de este trabajo es que el movimiento socioambiental ha tenido éxito, porque, una vez iniciados los trabajos del proyecto, los grupos interesados se estructuraron en una red de grupos afectados en la cual recibieron el apoyo de la sociedad civil para salvaguardar la fuente básica de su economía y su cultura.

Los resultados saltan a la vista. La empresa brasileña Odebrecht estableció acuerdos con el gobierno federal y estatal para la construcción de la hidroeléctrica, sin consultar a la población del municipio de Jalcomulco y sí dañando al ambiente con químicos tóxicos; sin embargo, los alcances hasta ahora han sido escasos porque, entre otros factores, una parte de la población y las organizaciones de la sociedad civil permanecen en acción y pendientes en la defensa del rio y de sus actividades.

El trabajo se articula en tres partes: la primera se remite a los antecedentes relativos al marco de la construcción de algunas presas en determinada parte del territorio mexicano, el desplazamiento y la reacción de los habitantes de las comunidades afectadas. En el segundo apartado se abordan algunos conceptos teóricos que ayudan a aproximarse con más objetivación y comprensión del tema de los movimientos o conflictos socioambientales, y el tercero se refiere a la relación estrecha entre la historia oral y los resultados empíricos del conflicto hasta este momento.

6 Como sugería la Escuela de los Annales "hay que comprender el pasado por el presente y el presente por el pasado, pero no analizar como historiadores el presente en sí mismo". Carlos Barros Guimerans, "Historiografía e historia inmediata: La experiencia latina de historia a debate (1993-2006)", Asociación de Historia Actual (HAOL), 9 (2006): file://C:/Users/Mary/Downloads/Dialnet-LaHistoriografiaYLaHistoriaInmediataLaExperienciaL-2195682.pdf. Pero retomando lo que señalaba M. Bloch de que la historia sea parte de un trabajo comprometido, plural y profesional, que aproveche la memoria activa de los actores intervinientes.

7 Mario Samper Kutschbach, "Los usos de la historia para construir proyectos de futuro", Revista de Historia (Costa Rica) 70 (julio-diciembre 2014). 
Durante el gobierno del presidente Porfirio Díaz, a principios del siglo XX, cuando México se incorporó al carro del progreso, según el ideal del liberalismo, se construyeron presas como la de Necaxa, en Puebla y Taximay, en Hidalgo, las cuales sirvieron para el abastecimiento de las zonas mineras y de las ciudades cercanas a dichas presas, que ya contaban con un desarrollo industrial y comercial como las ciudades de Puebla, México y Pachuca.

Para la instalación de las cortinas y la maquinaria en los cauces de los ríos, fue necesario desplazar varias congregaciones y pueblos que, aunque no estaban de acuerdo, no pusieron mucha resistencia, pues temían la represión del gobierno; además, les concedieron otras tierras, donde les reubicaron sus huertas, casas y terrenos de cultivos; por cierto, fueron territorios nuevos donde tuvieron que recomenzar sus actividades campesinas.

Después del movimiento armado de la revolución mexicana, la construcción de las represas o presas hidroeléctricas en México se ha dado por etapas y ha estado ligada a las distintas políticas que comprenden las necesidades económicas y sociales del país.

Para comenzar, la etapa que marca la expansión de México en este rubro estuvo dirigida por un sector de élite norteña, encabezado por Venustiano Carranza (1917-1920), Álvaro Obregón (1920-1924), Plutarco Elías Calles (19241928) y Abelardo Rodríguez (1932-1934). Durante estos gobiernos se impulsó la modernización de la agricultura en el árido norte, que padecía escasez y sequias; por ello, se construyeron varias presas que concentraron el agua para regular y mantener el riego de los campos agrícolas. Pero no solo se modernizó el norte con este tipo de obras, sino también el centro y occidente del país, debido a que el desarrollo industrial, agrícola, ganadero y comercial impulsó la movilización de la gente del campo a ciudades como Monterrey, Guadalajara, Querétaro Puebla, México y Veracruz, centros que incrementaron su población y con ello los requerimientos de servicios básicos.

De ahí que Luis Aboites en sus libros; El agua de la Nación y La decadencia del agua de la nación ${ }^{8}$ indica que hay dos etapas en la edificación de obras hidráulicas. La primera, que va de 1926 a 1946, cuando se construyeron importantes obras de ingeniería para controlar el agua en presas y apoyar el riego del árido norte, producir electricidad y abastecer de agua a las ciudades de mayor demanda por el desarrollo económico en el centro del país. En la segunda etapa, de 1946 a 1976, se construyeron presas multipropósito que cubrían el riego, el abasto a las poblaciones, la generación de electricidad, el control de avenidas

8 Luis Aboites Aguilar, La decadencia del agua de la nación. Estudio sobre la desigualdad social y cambio político en México, segunda mitad del siglo XX (México, D.F.: El Colegio de México, 2009). 
y la prevención de inundaciones. ${ }^{9}$ Posterior a este periodo, la construcción de obras fue menor, dada la situación económica del país. Sin embargo, las de gran envergadura se habían hecho en las etapas señaladas, y justo en los cauces de los ríos de mayor caudal; incluso estos dos periodos se reconocen como las etapas de la gran hidráulica, es decir, las obras que prosiguieron a este período, en general, han sido de menor tamaño.

Las primeras presas potenciaron el desarrollo agrícola y ganadero de las regiones que padecían escasez y sequias, fundamentalmente en las cuencas del norte del país, a la vez que satisfacían la demanda social y económica del bajío y el altiplano. Por mencionar unas cuantas tenemos la de San Bartolo en Durango, Abelardo Rodríguez en Tijuana BCN, la Venustiano Carranza en Coahuila o la Requena en Hidalgo. En los estudios que se han realizado sobre este tema se profundiza más sobre la estructura y costos de las obras, pero no sobre el desplazamiento de grandes poblaciones ni las afectaciones al ambiente ni los costos sociales, porque el norte era una zona escasamente habitada y porque el propósito de los proyectos hidroeléctricos era impulsar su desarrollo económico y movilizar población hacia aquella zona.

Las presas del segundo período, en su mayoría, se construyeron en los cauces de los grandes ríos del país, porque los propósitos, como se indicó, eran múltiples y respondían a una etapa de mayor demanda en el control de los recursos para la economía mexicana en auge. Por ejemplo, en 1955 se construyó la presa Miguel Alemán, en el cauce del río Tonto entre Oaxaca y Veracruz, en territorios indígenas mazatecos: 22.000 personas de este grupo sufrieron un desplazamiento forzado porque no querían abandonar sus tierras, pero tuvieron que hacerlo forzadas, pues en la agresión les incendiaron sus casas. ${ }^{10}$

Posteriormente en territorio oaxaqueño, muy cerca de la presa Miguel Alemán, se promovió "la construcción de la presa Cerro de Oro -en el río Santo Domingo- para la que se desalojaron a 26.000 campesinos de origen indígena Chinantecos"11 que hasta 2010 seguían un proceso legal para obtener indemnizaciones por las tierras de cultivo perdidas, puesto que el gobierno, en alguno de sus niveles, les ocultaba la información pública e, incluso, se acusaba de diversos

9 Estas etapas coinciden con el proceso de institucionalización ligada al control y reglamentación de los recursos básicos de algunos organismos como la Comisión Nacional de Irrigación (CNI) en 1926, -y respondió fundamentalmente a los intereses del riego- cuando las políticas cambiaron hacia el modelo de desarrollo de la revolución verde. En este periodo, justo en 1946, se formó la Secretaría de Recursos Hidráulicos (SRH), a la que le correspondió la reestructuración de la política en el manejo del agua que fue sustituida en 1976 por la Secretaría de Agricultura y Recursos Hidráulicos (SARH), cuya necesidad fue darle más atención al sector agropecuario, antecedente de la actual Comisión Nacional del Agua.

10 Tribunal Permanente de los Pueblos (TPP), Pre-Audiencia sobre Presas, Derechos de los Pueblos e Impunidad. Mesa de devastación ambiental y derechos de los pueblos. Capítulo Mexicano del Tribunal Permanente de los Pueblos. Dictamen Final, (Temacapulin, Jalisco: TPP) Disponible en: http://www.tppmexico. org/wp-content/uploads/2013/04/Dictamen_final_preaudienciaPresasTPP.pdf

11 Ibid. 
delitos a sus líderes sociales para desarticular la protesta. ${ }^{12}$ De igual forma se dieron los acontecimientos en los territorios de Chiapas, en las márgenes del río Grijalva, entre 1964 y 1974, cuando se edificaron las grandes presas Netzahualcóyotl y la Angostura, ya que para la instalación de las cortinas y la maquinaria en el gran cauce del río, fue necesario desplazar a habitantes de varias congregaciones y pueblos oaxaqueños, veracruzanos y chiapanecos, porque sus tierras serían inundadas y sus cultivos destruidos.

Los grupos étnicos y campesinos agraviados no estaban de acuerdo y pusieron cierta resistencia para hacer que se respetaran sus espacios, pero no contaron con el apoyo suficiente y temían que el gobierno los reprimiera, como ya lo había hecho con los mazatecos, por eso no tuvieron otra alternativa que reubicarse en otras tierras donde empezaron a roturar los campos para sus huertas y terrenos de cultivos y todas las actividades propias para tener su casa y su espacio de reproducción social. En la década que comprende de 1960 a 1970 estos sectores sociales no representaban problemas de interés público. Aunque ya comenzaban a generarse algunos estudios de carácter ambiental como los de Rachel Carson, Richard Adams, Elinor Melville, entre otros, este discurso académico con repercusiones político-sociales no era accesible a otros espacios, por lo tanto, la sociedad no tenía conocimiento de los daños ambientales que la construcción de presas ocasiona.

Hoy existen diversos estudios centrados en las afectaciones ambientales que ocasionan las obras hidroeléctricas en los territorios. Unos aplican el enfoque de la economía ecológica; otros, el de las estructuras de poder, los movimientos sociales, la racionalidad económica o la defensa de los pueblos indígenas y el aspecto emocional y cultural, es decir, las miradas son diversas y los medios potencializan su conocimiento y, por lo tanto, sus prácticas.

En este caso, se considera necesario partir de los conceptos propuestos de forma conjunta por Joan Martínez Alier y James O'connor, quienes, desde la economía ecológica, hablan de las externalidades económicas y sociales que se producen cuando un territorio es afectado por la construcción de una presa y que se manifiestan en la pérdida de bienestar social de sectores ajenos a las empresas que las construyeron y pusieron en operación, ${ }^{13}$ puesto que la modificación del territorio comienza desde los estudios de suelo para construcción, la deforestación que conlleva, así como otras maniobras tales como la fractura de los cerros para hacer túneles a fin de desviar y conducir del agua. Estas prácticas han ocasionado la inundación de un número indefinido de hectáreas de tierras, según el tamaño de la obra; ello conduce a la pérdida del potencial de la flora y

12 Mario Alberto Velázquez García, Las luchas verdes. Los movimientos ambientalistas de Tepoztlán, Morelos, y el Cytrar en Hermosillo, Sonora (Sonora, México: El Colegio de Sonora, 2009).

13 Joan Martínez Alier, "Los conflictos ecológicos distributivos y los indicadores de sustentabilidad" en: Revista Iberoamericana de Economía Ecológica, 1, (2004): http://www.raco.cat/index.php/Revibec/article/ view/38278/38152 
fauna, de los recursos maderables de los bosques, pastizales y cultivos, así como la reducción potencial de peces, camarones y otros habitantes de los ríos, lo que contribuye al desplazamiento de los grupos humanos que son despojados de un territorio que es su medio de supervivencia.

En los territorios donde anteriormente se construyeron presas de gran envergadura, la población se desplazaba hacia otros sitios $\mathrm{y}$, aunque hubo resistencia, fue menos observada y más controlada por las instituciones gubernamentales. Hoy la situación es diferente, puesto que los proyectos hidroeléctricos, en el caso mexicano, tienden a desarrollarse en espacios con menos potencial hidráulico, en contraste con la primera etapa. Asimismo, tienen visos de ser distintos, porque hay una mayor participación de otros actores sociales -llámense organizaciones civiles, académicas, empresariales o de grupos en red-, así como de una mayor concientización de lo que representa el ambiente; también se ha generado un marco legislativo que hoy defiende a los grupos indígenas o a los sectores vulnerables ante las practicas agresivas del neoliberalismo. ${ }^{14}$

Lo importante es tratar de explicar cómo el sistema capitalista, en su fase neoliberal actual, representa una forma de mayor depredación por las acciones coercitivas que se ejercen en aquellas demarcaciones con más población marginada y menos posibilidades de sobrevivencia, o en los espacios en donde los ríos y su acceso estratégico promovieron un desarrollo económico basado en la pesca, la agricultura de plantación y el ecoturismo. Estas actividades peligran al ser afectadas y con ello los medios de vida de la población local y regional, lo que ha ocasionado un movimiento social para proteger su subsistencia.

Por esta razón se han realizado variados estudios que enfatizan no solo la vulnerabilidad de los sectores de población agraviados en sus territorios y sus diversas actividades, sino también la afectación ambiental, económica y social que producen estas obras.

La población agraviada se ha organizado y mantenido en sus protestas desde el momento en que se hicieron pruebas sobre la factibilidad de la construcción en el territorio afectado o en cualquiera de las fases de construcción. De ahí que las protestas se mantienen, diluyen o tienen compases distintos, dependiendo de la fortaleza de los grupos de acción y de las conexiones que establezcan con otros movimientos sociales, bien sea por la identidad o por el conocimiento: lo importante es brindarse apoyo en la defensa de sus recursos, frente a la depredación de las grandes empresas capitalistas.

14 Convenio 169 de OIT, que protege los derechos de las comunidades indígenas ante la desaparición de sus sistemas de creencias, su idioma y la vulnerabilidad de su territorio, a esto se suma el convenio 107 sobre derechos de los pueblos indígenas y tribales de la OIT, la Declaración de la ONU sobre los derechos de los pueblos indígenas de 2007. "La verdad sobre el proyecto hidroeléctrico", video de: Contra el PH Diquis, 1 de agosto de 2010, disponible en: https://www.youtube.com/watch?v=x1uuIYNaOls 
Para empezar, es importante tener claro que el término conflicto significa choque o golpe, es decir, define una situación antagónica entre distintos actores -personas, grupos, organización social o institución- que persiguen intereses políticos o económicos contrarios, lo que los coloca en situación de enfrentamiento, confrontación y lucha consciente en la búsqueda de metas económicas y políticas, de acuerdo con la teoría marxista. En cuanto al término ambiental, este hace referencia a todo proceso que expresa una relación entre la naturaleza y la cultura, de ahí que el conflicto ambiental se produce cuando hay un proceso de apropiación y transformación de la naturaleza debido a la incorporación de los sistemas tecnológicos que afectan directamente al ser humano. ${ }^{15}$

Autores como Bookchin, ${ }^{16}$ Sabatini, ${ }^{17} y$ Walter ${ }^{18}$ hacen una tipología de conflictos diferenciándolos, por lo general, en ambientales, socioambientales y distributivos, de acuerdo con las diferencias en las afectaciones que el ser humano hace de un determinado espacio ecológico con las innovaciones tecnológicas y las formas en que impactan a los grupos humanos, aunque también por la intensidad en que se manifiestan las acciones entre los actores implicados y el grado de afectación al medio ambiente.

Una característica de los conflictos ambientales es que los actores antagónicos generan un problema de carácter ecológico produciendo afectaciones o impactos dañinos a quienes viven en el espacio modificado por las tecnologías. Mientras que los conflictos socioambientales se presentan cuando los actores implicados se disputan la distribución y uso de los recursos naturales con pobladores de un territorio determinado, y afectan la propiedad o posesión sobre los recursos naturales que necesitan las personas, comunidades y naciones para producir bienes y servicios que satisfagan sus necesidades, ${ }^{19}$ por lo cual surge la protesta que consiste en una denuncia contra los entes causantes de los daños ambientales.

De ahí que, específicamente, los conflictos de carácter socioambiental se presentan cuando hay relaciones de choque y confrontación, debido a las dificultades en la interacción social, la falta de diálogo y la escasa o nula participación de la población local en las decisiones públicas. Sabatini, ${ }^{20}$ retomando a

15 Jorge Riechmann y Francisco Fernández Buey, redes que dan libertad: Introducción a los nuevos movimientos sociales (Barcelona, España: Editorial Paidós, 1994) y Velázquez García.

16 Stephen Bookchin, Ecología social y comunalismo (Oakland, EE. UU.: AK Press, 1993).

17 Francisco Sabatini, y Claudia Sepúlveda L., Conflictos ambientales: Entre la globalización y la sociedad civil (Santiago, Chile: Publicaciones CIPMA, 2002).

18 Mariana Walter, "Conflictos ambientales, ecológico distributivos, de contenido ambiental. Reflexionando sobre enfoques y definiciones”, CIP ECOSOCIAL (ECOS), 6 (febrero-abril 2009): http://www.fuhem.org/ media/ecosocial/File/Boletin\%20ECOS/Boletin\%206/Conflictos\%20ambientales_M.WALTER_mar09 final.pdf

19 Walter.

20 Sabatini y Sepúlveda. 
Bookchin, refiere que se trata de conflictos de orden más social y político que técnico. ${ }^{21}$ De hecho, el conflicto que se presenta en la zona de estudio tiene un carácter socioambiental en el que están implicados el Estado, la empresa extranjera Odebrecht y la sociedad civil, que incluye activistas, sectores académicos, organizaciones no gubernamentales y la población participativa.

Para lograr una mayor comprensión del conflicto socioambiental en Jalcomulco es importante retomar los estudios relacionados con los movimientos sociales, porque el referido conflicto motivó a la población a movilizarse para defender su espacio de trabajo. Jorge Reichmann y Francisco Fernández apuntan que un movimiento social es un agente colectivo que interviene en el proceso de transformación social promoviendo cambios u oponiéndose a ellos. De modo que todo movimiento social como agente colectivo persigue el objetivo de provocar, impedir o anular un cambio social, obrando con cierta continuidad, con un alto nivel de integración simbólica y un nivel bajo de especificación de roles, con formas de acción y organización variables. ${ }^{22}$ Desde esta definición se puede hacer referencia a los movimientos que han sido importantes en la transformación social del mundo contemporáneo.

Alberto Melucci ${ }^{23}$ argumenta que un movimiento social es una forma de acción colectiva que apela a la solidaridad de grupo para disputar, contra otros, los mismos recursos. Alain Touraine ${ }^{24}$ considera que un movimiento social es "una conducta colectiva organizada de un actor luchando contra su adversario por la dirección social de la historicidad en una colectividad concreta, sujeta a la implementación de valores culturales" ${ }^{25}$ que les da identidad en contra de los intereses e influencias de un enemigo definido en términos de relaciones de poder.

Los tres autores citados coinciden en que existe una génesis común de todo movimiento social: la acción colectiva, es decir, la acción que motiva a los individuos a actuar en aras de un beneficio colectivo; la diferencia entre ellos estriba en que mientras uno habla de roles sociales, otro insiste en la existencia de solidaridades y el tercero se centra en la identidad de los grupos sostenida por elementos culturales. En este sentido, el movimiento social es la movilización voluntaria de hombres y mujeres en torno a una causa, intereses, emociones y esperanzas, en la que los sujetos no se limitan a protestar, sino que establecen sus propias formas de organización. ${ }^{26}$

21 Velázquez.

22 Riechmann y Fernández Buey.

23 Alberto Melucci, Acción colectiva, vida cotidiana y democracia (México, D.F.: El Colegio de México, Centro de Estudios Sociológicos, 1999).

24 Alan Touraine, "Los movimientos sociales", Revista Colombiana de Sociología (Colombia) 27 (2006): http://www.revistas.unal.edu.co/index.php/recs/article/view/7982/8626

25 Touraine, 255.

26 Jorge Alonso, Repensar los movimientos sociales (México: CIESAS, Publicaciones de la Casa Chata, 2013). 
La acción colectiva es el denominador común de los movimientos sociales, ${ }^{27}$ resultado de intenciones, recursos y límites, con una orientación construida mediante relaciones sociales al interior de un sistema de oportunidades y restricciones. ${ }^{28}$ Entonces, en las acciones colectivas se destacan los vínculos que propician la creación de un agrupamiento que define activamente intereses comunes que mantienen la solidaridad en busca de actos reivindicatorios; este es el principal recurso y, con frecuencia, el único del que dispone la mayoría de la gente para enfrentarse a su adversario. De modo que los movimientos sociales son un tipo especial de acción colectiva, donde es clara la actuación voluntaria a favor de una causa que da identidad a sus miembros durante la disputa y en la que están identificados los adversarios, ya que para luchar es necesario saber en nombre de quién, contra quién y sobre qué terreno se lucha. ${ }^{29}$

Algunos movimientos promueven la acción colectiva directa o de protesta para ejercer presión por situaciones problemáticas que atentan contra la sobrevivencia y la seguridad de las personas. Estos han trastocado los espacios vitales sin contribuir a resolver las necesidades reales de amplios sectores sociales, sino, más bien, aumentando el hambre, el desempleo y la pobreza e inclusive el desalojo y la inmigración a los sectores más desprotegidos, sin olvidar que otros sectores también son afectados. ${ }^{30}$ Los movimientos sociales ambientalistas surgen a partir de la inconformidad de la ciudadanía contra quienes abusan del medio ambiente, y se fortalecen con el movimiento democrático y los acuerdos paralelos sobre el trabajo y la salud, en los tratados comerciales internacionales. Mientras que los conflictos de distribución ecológica y movimientos de resistencia surgen por la desigual titularidad de los recursos naturales, los servicios ambientales se entrelazan algunas veces con conflictos de distribución económica en las comunidades. Los movimientos de resistencia están trabajando por la sobrevivencia de las comunidades.

\section{La presa hidroeléctrica y los actores intervinientes en el movimiento}

El gobierno Federal, a través Comisión Nacional para el Uso Eficiente de la Energía, realizó hace más de 15 años un diagnóstico para medir el potencial hidroeléctrico del estado de Veracruz, y el posible aprovechamiento de los rios en cuanto a la generación de energía y el abasto de agua potable a las poblaciones.

El estado de Veracruz cuenta con 6 cuencas hidrográficas que son: Actopan, la Antigua, Nautla, Tecolutla, Cazones y Tuxpan, en las cuales se planeó llevar a cabo 112 proyectos hidroeléctricos, entre los que se consideraron la mini

27 Sidney Tarrow. El poder en movimiento: los movimientos sociales, la acción colectiva y la política (España: Alianza Editorial, 1997).

28 Melucci.

29 Alonso. Touraine.

30 Riechman y Fernández Buey. 
presa del Naranjal, el trasvase del río Pánuco a Monterrey y el proyecto de propósitos múltiples de Xalapa. Este último contempla la afectación del río los Pescados en la zona de Jalcomulco. Dicho proyecto se inició en octubre de 2013, cuando Marcelo Odebrecht, dueño de la empresa que lleva su apellido, se reunió con el Presidente Peña Nieto, para dar comienzo formal a la construcción de la presa. Ya contando con la autorización federal, se iniciaron los trabajos de exploración, para lo cual se llevó maquinaria al ejido El Tamarindo en las márgenes del río los Pescados.

\section{Características del proyecto hidroeléctrico de Jalcomulco}

Antes de señalar las características del proyecto es importante destacar la localización geográfica de Jalcomulco y las actividades a las que se dedica la población que sería más afectada por la construcción de la presa. La zona de Jalcomulco se ubica sobre el río Los pescados, el cual forma parte de la cuenca del río la Antigua, caudales que descienden de la Sierra Madre Oriental y el sistema volcánico transmexicano, representado por el Cofre de Perote y el Pico de Orizaba en el centro del estado de Veracruz.

Este poblado es cabecera municipal y cuenta con más de 5.000 habitantes, ${ }^{31}$ que viven en un clima cálido-húmedo, con una altitud que va de los 200 a los $900 \mathrm{msnm}$, una temperatura anual promedio de $24^{\circ} \mathrm{C}$ y una precipitación de $1,018 \mathrm{~mm}$. Jalcomulco es un territorio tranquilo, cuya población se dedica a distintas actividades productivas que se realizan aprovechando las corrientes del río Los pescados, los suelos fértiles y el clima. La principal fuente de ingresos de la población de Jalcomulco y sus alrededores es, principalmente, el cultivo de la caña de azúcar, mango, limón, café y otros frutos que ahí se producen, a lo que se suma la pesca -de pez bobo, mojarra, camarón, acamayas o langostinos-, que forma parte de la cocina regional, la artesanía -cestería, productos de barro, jícaras, lianas, bejuco y palma- ${ }^{32}$ y el turismo, que en los últimos quince años se convirtió en una "mina de oro". Incluso la inserción del llamado ecoturismo marcó un cambio en las otras actividades productivas, porque se incrementó el empleo en los servicios ligados al turismo, así como el consumo de los productos locales ${ }^{33}$ y el apogeo del área gastronómica y artesanal.

Cabe señalar que el auge de la actividad ecoturística en Jalcomulco se dio en respuesta a la tendencia global para el desarrollo de deportes extremos y actividades de entretenimiento relacionadas con estos, de ahí la inversión de

31 Plan Municipal de Desarrollo de Jalcomulco (2014-2017) (México, 2014).

32 Como don Anselmo Lobato, campesino y pescador de Jalcomulco, quien mientras realiza su guardia en el campamento, elabora con bejuco, hilaza, chaquira, semillas y plumas "atrapasueños", que luego vende a otras personas que tienen sus comercios de artesanía para el turismo en Jalcomulco. Entrevista con don Anselmo Lobato, 15 de mayo de 2015, Campamento El Tamarindo, Jalcomulco, Veracruz, México.

33 Plan Municipal de Desarrollo de Jalcomulco (2014-2017) (México, 2014). 
capitales nacionales e internacionales en lugares óptimos para la explotación, dado su potencial de riqueza en recursos naturales aprovechables para el desarrollo del ecoturismo. Muestra de ello es que en el área centroamericana -para este caso- se encuentran desarrollos alternativos como: los Arenales, Nosara o Playa Tamarindo en Costa Rica, o el Lago Nicaragua y el Archipiélago de Solentimane en Nicaragua, o el propio Jalcomulco en México, todos dirigidos al consumo "turístico", en su mayoría, norteamericano y europeo.

Para la población de Jalcomulco y sus alrededores, el río significa un modo de vida porque con esta corriente se riegan los cultivos de sus tierras, se pescan productos para la gastronomía local que, junto con el aprovechamiento del río en tiempos de calor, atraen al turismo a esta zona.

La condición de la corriente del río los pescados, que recorre las barranca de Jalcomulco, Chicabaxtla, Tuzamapam, Barranca Grande y los cerros de Pizaltepec y Acuamali, entre otros atractivos, determinó que el desarrollo del turismo de aventura -que incluye kayakismo, escalada, rapel, campamentos, senderismo, rafting y natación ${ }^{34}$ alcanzó a promover el auge de 32 empresas turísticas ${ }^{35}$ de capital local, regional y nacional que tiene una afluencia anual superior a los 50.000 visitantes en promedio, lo que se traduce en importantes ganancias para quienes trabajan en las empresas, restaurantes y tiendas -artesanías, frutos y producto locales- que dan servicio todo el año y preferentemente en temporadas vacacionales.

De acuerdo con la Gaceta Legislativa No. $142,{ }^{36}$ en Jalcomulco se pretende desarrollar el proyecto hidroeléctrico de Propósitos Múltiples Xalapa, con la construcción de la presa en la cuenca del río La Antigua, para suministrar agua a la ciudad capital estatal, generar energía eléctrica a industriales e irrigar tierras agrícolas ${ }^{37}$ Es cierto, la ciudad de Xalapa padece un déficit del recurso; pero: ¿cómo garantizar que el agua llegue a la población capitalina, si la altura promedio es de $1.417 \mathrm{msnm}$ ?, mientras que la altura de Jalcomulco es menor a la altura de $500 \mathrm{msnm}$; es decir, se tendría que gastar en el bombeo del agua,

34 Ibid.

35 Muchas de estas empresas se localizaron en el camino hacia Jalcomulco y en el poblado mismo: las siguientes: 1) Raft México, 2) Expediciones México Verde, 3) Jalco-Expediciones, 4). Ecoturismo en la Aldea, 5). Villa Pinome, 6). Aventurec, 7. Aventuras Sin Límites Expediciones, 8) Green Eco Ressort, 9) Amigos del Río, 10) Yolotl- Corazón del Río, 11) ProffesionalAdventureTeam, 12) Rancho el Macarrón, 13) Cotlamani-Hotel, 14) SelvAzul, 15) Desafiando-Expediciones, 16) Cañon de Zicatla, 17). Xploraciones-Río Montaña, 18) Rioxanecatl, 19) Huitzilapan Expediciones, 20) Ecco. Sport (n.1994), Río Aventura, 21) Quinto Sol, 22) Expediciones Selectas, 23) La Tribu, 24) Eccotravel, 25) Veredas Selectas, 26) Aventura Vertical, 27) Arcoiris Sport- Fishing, 28) Aldea Rodaaventura, 29) Río Salvaje. De estas se sabe que Aventuras Sin Límites Expediciones, Jalco Expediciones, Huitzilapan Expediciones, Río Salvaje y México Verde, son cinco empresas locales que participan en el movimiento. Observación directa en la zona, abril y mayo de 2015.

36 Gobierno del Estado de Veracruz (Vercruz, México: marzo 2013). Disponible en: http://www.legisver.gob. $\mathrm{mx} /$ transparencia/FraccionXXXVI/ORDEN_DEL_DIA_142.pdf

37 Noé Zavaleta, "Comuneros de Veracruz frenan al "Goliat" brasileño", Proceso, 2 de mayo de 2014: http:// www.proceso.com.mx/371199/comuneros-de-veracruz-frenan-al-goliat-brasileno-2 
porque la presa quedaría bastante abajo, lo que implicaría invertir en dos cortinas o más, y resultaría bastante oneroso. Por lo que, de acuerdo con la racionalidad económica, se pensaría que la producción de energía sería para venderla a grandes empresas consumidoras, por ejemplo, a las grandes industrias; entonces, la construcción de la presa responde más a una necesidad extra regional, que al abastecimiento de la ciudad capital. Más bien apunta a los acuerdos subrayados en el plan Puebla Panamá.

El costo de la presa sería de 7 mil 400 millones de pesos, de los cuales $72 \%$ lo aportaría la empresa brasileña Odebrecht, con un capital de 5 mil 328 millones de pesos, mientras que la Comisión del Agua del Estado de Veracruz (CAEV) aportaría 28\%, es decir, 2 mil 072 millones. Ello supone un manejo privado del agua, porque, además, la empresa se encargaría de la administración del recurso de acuerdo con la inversión financiada y lo pactado con el gobierno. ${ }^{38}$

La Declaratoria de Centinelas del Río la Antigua (20-I-2015) indica que, de ser edificada, las dimensiones de la cortina serían de 100 metros de altura por 700 metros de ancho y contaría con una planta generadora de 70 megavatios de electricidad. ${ }^{39}$ Para conseguir realizar la obra se inundarían 400 hectáreas de terrenos planos y accidentados donde hay cultivos de caña de azúcar, mango, limón, papaya, café, otros frutales y ganado. Estos productos son de autoconsumo y de mercado, y serían afectadas lo mismo que varias poblaciones de los municipios de Ayahualulco, Ixhuacán de los Reyes, Cosautlán, Coatepec, Tlaltetela, Teocelo, Jalcomulco, Apazapan, Emiliano Zapata y La Antigua ${ }^{40}$-véase mapa 2-. Cabe señalar que estas poblaciones son de mayor concentración de habitantes y con un desarrollo importante en su economía. La inundación destruiría flora y fauna en el espacio ocupado por la cortina, el trasvase y la maquinaria que abre o cierra la presa. Por otra parte, estos municipios están al pie de monte descendiendo del Cofre de Perote, terreno que en parte se reconoce como geológicamente inestable, debido a que en los últimos 70 años han ocurrido tres sismos de más de 7 grados en la escala de Richter, ${ }^{41}$ lo cual constituye un factor de riesgo para la construcción de la presa.

38 "Construirá Odebrecht presa en Veracruz", La Jornada, Veracruz, 15 de marzo de 2013: http://www.jornada.unam.mx/2013/03/15/estados/040n5est

39 “Odebrecht: una amenaza que corre por el río", Forbes México, 5 de agosto 2014: http://www.forbes.com. $\mathrm{mx} /$ odebrech-una-amenaza-que-corre-por-el-rio/

40 "Le avanzó Odebrecht a Presa en Tamarindo: Alcalde", La Politica.mx, 22 de enero de 2014: http://www. lapolitica. $\mathrm{mx} / \mathrm{p}=202627$

41 "Presa Jalcomulco, un proyecto en contra del bien común", video de La Jornada Veracruz, 5 de marzo de 2014: https://www.youtube.com/watch?v=iHyPsjO0INw 


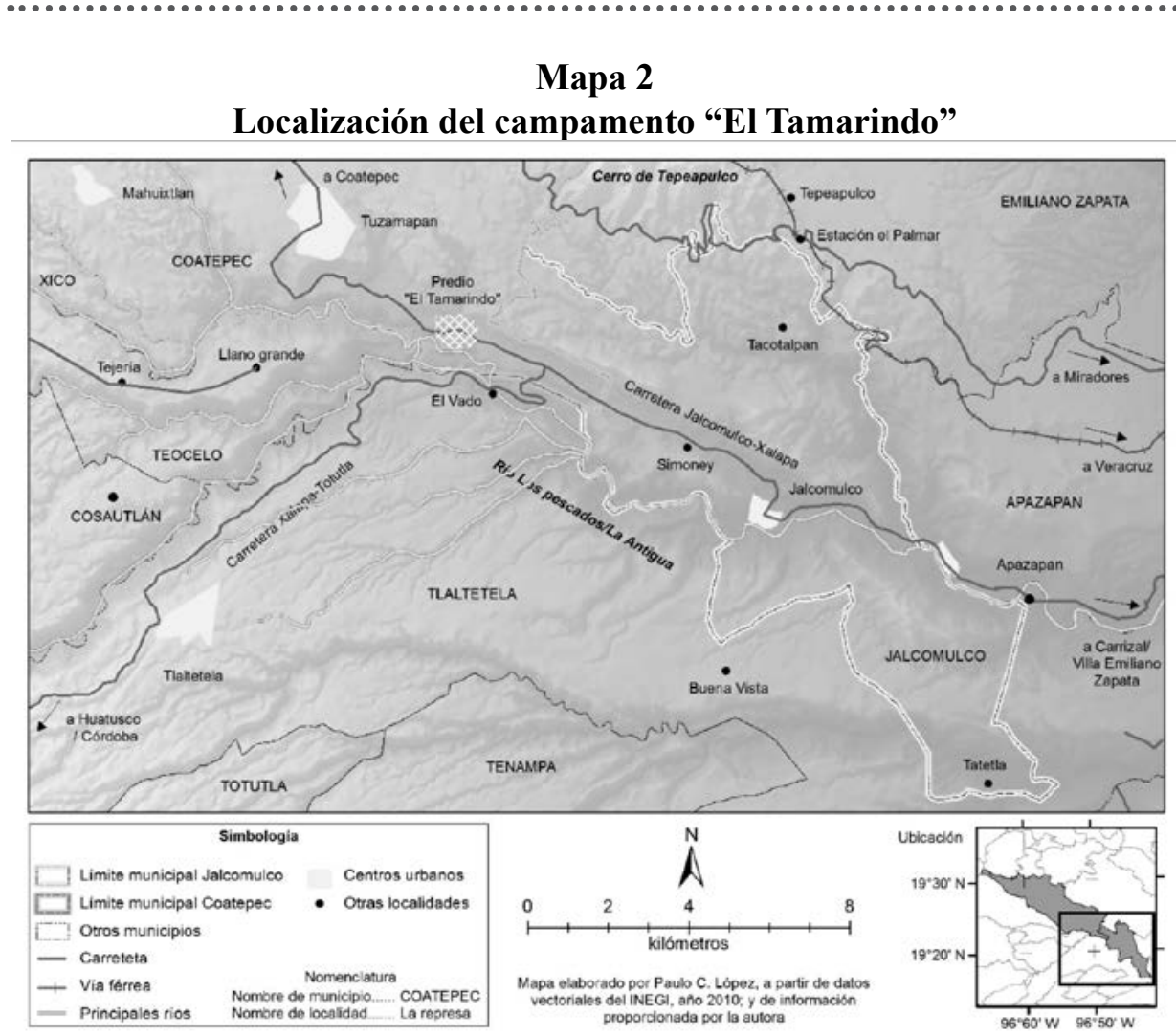

Fuente: Elaboración a partir de datos del INEGI, (México. 2010).

La concretización del proyecto sobre el río Los Pescados en la zona de Jalcomulco se inició en octubre de 2013, cuando Marcelo Odebrecht, dueño de la empresa, se reunió con el presidente Peña Nieto para dar comienzo formal a la construcción de la presa y, ya contando con la autorización federal, se iniciaron los trabajos de exploración, para lo cual llevaron maquinaria al ejido Tamarindo en la ribera del río Los Pescados. Este ejido pertenece al municipio de Tuzamapan y colinda con Jalcomulco, Coatepec y Cosautlán; pero como la afectación mayor sería para Jalcomulco, sus habitantes se movilizaron rápidamente para detener el proyecto.

Aunque existían rumores de que iban a construir una presa en esta zona, su población carecía de información y datos concretos de cómo, cuándo y dónde exactamente se construiría, por eso cuando llegó la maquinaria al ejido Tamarindo a ocupar terrenos sin haber solicitado permiso a sus propietarios, ${ }^{42}$ se socializó la noticia a través de las redes sociales preexistentes, porque en esta región hay una herencia ejidal que rescata las prácticas comunales relacionadas con la

42 Entrevista a Lúcido, 26 de mayo de 2015, Campamento El Tamarindo, Jalcomulco, Veracruz, México. 
producción agrícola y ganadera, así como también de organizaciones vecinales y de ecoturismo; este último, por cierto, es el que mantiene una mayor relación con los medios de comunicación. Estas redes y organizaciones generan identidad y unidad, por tanto, posibilitan la organización de grupos y, a pesar de que el gobierno no les proporcionó información clara y suficiente, la identidad de grupo fue la base para iniciar la oposición.

El mismo día de la llegada de la empresa Odebrecht con sus trabajadores al terreno del ejido El Tamarindo, donde iniciaron la exploración con ventonita, los grupos opositores -entre los que estaban campesinos, ejidatarios, cañeros, guías de río y otros pobladores de toda la zona de Jalcomulco- ya estaban presentes y expulsaron a los trabajadores de la empresa por no contar con permisos para la realización de los trabajos previos a la construcción de dicha presa; sin embargo, una semana después, el corporativo regresó con permisos falsos de Conagua y Semarnat, pero se enfrentó nuevamente a la oposición de la resistencia, quienes buscaron apoyo de los pueblos afectados y, ya reunidos con mayor fuerza, se armaron de valor y cerraron la autopista en la caseta de La Antigua, cercana al Puerto de Veracruz, por donde concurre un tráfico vehicular intenso. De esa forma, consiguieron que casi toda la maquinaria y los químicos utilizados para los trabajos de exploración fueran retirados del predio; sin embargo, la empresa no ha desistido de su empeño de hacer la presa, pues cuenta con el apoyo de las autoridades y organismos estatales y federales. Por tal motivo, los manifestantes se organizaron para permanecer en un campamento nombrado "El centinela", con el propósito de vigilar la entrada del predio en donde se quiere edificar la presa.

¿Qué ha hecho la población para construir y mantener su fuerza como resistencia en la defensa del río? Se ha integrado y organizado en un movimiento de resistencia, realiza asambleas y talleres informativos con el propósito de hacer conciencia de los daños que ocasionaría la construcción de una presa a sus tierras, a sus actividades productivas, turísticas, culturales y a su vida cotidiana.

La mayoría de la población, concentrada en el trabajo campesino o en otras actividades relacionadas con el turismo, carecía de información y en estos talleres le han dado a conocer los problemas que padecen otras zonas por la construcción de hidroeléctricas, a través de pláticas y enseñanzas que incluían diversas manifestaciones y experiencias vividas como las inundaciones, desalojo y otros riesgos que se padecen en los lugares donde se han construido presas. La gente, al enterarse "de la catástrofe ambiental en el río los Pescados", 43 la cual tendrá fuerte impacto social y económico en habitantes de la zona-despojo de sus propiedades, sus actividades agropecuarias, turísticas y comerciales y de recreación- se motivó y se incorporó al movimiento y al campamento, ya que

43 "Hidroeléctricas son sinónimo de catástrofe ambiental: activistas", AVC Noticias, 1 de diciembre, 2014: http://www.avcnoticias.com.mx/resumen2.php?idnota=171794 
está consciente sobre el costo social, económico, cultural y ambiental que le acarrearía la construcción de la presa, por el apego que tiene a los recursos naturales y a la cultura del lugar, que forma parte de su vida cotidiana. Sin embargo, hay algunos grupos empresarios que se oponen, porque lo que los mueve es el lucro o la ganancia como eje empresarial; es decir, se puede observar una visión conservacionista que los anima a invertir en tierras y desarrollo turístico, sin importarles los costos ambientales. Por lo menos eso es lo que dejan ver varias empresas externas dedicadas a la explotación turística.

Parte de la población se mantiene al margen y no participa por temor, desconocimiento o por el individualismo que prevaleció en personal empleado en el sector terciario y que solo depende de su fuerza de trabajo; a esto se suman las personas adultas mayores parceleras, cuyas familias están ausentes del campo. Estas han visto afectados sus recursos por la cementera de Apazapan, que construyó una represa para su funcionamiento y, a pesar de que absorbió la mano de obra local, los químicos contaminantes que expande de su industria afectaron la producción local, como lo refiere uno de los entrevistados "que a causa de los polvos de la cementera, los cultivos de papaya enfermaron y se acabaron, cuando antes las papayas, grandes y preciosas" ${ }^{44}$ eran un símbolo de la feracidad de la región, por lo consiguiente ya no tienen interés en lo que suceda en el campo y solo les queda sobrevivir.

La acción colectiva se identifica con los propósitos de defender su tierra, sus costumbres y su trabajo, con lo que han logrado mantener una organización que comenzó "acudiendo a las distintas poblaciones implicadas en las afectaciones que dejaría la presa, exponiéndoles todos los riesgos ambientales, sociales y económicos que ocasionaría una construcción de esa magnitud". ${ }^{45}$ Lo que quiere decir es que las personas afectadas son conscientes y conocedoras de las externalidades del proyecto y conforman el marco de la acción colectiva del movimiento, al conjuntar las intersubjetividades que identifican a los pobladores y pobladoras, quienes tienen apego al lugar, ya que eso determina su participación en el campamento. ${ }^{46}$

Todo lo anterior permite observar que hasta hoy es un movimiento más o menos exitoso, en el cual se observa que el campamento de vigilancia está integrado por diversos grupos que hacen guardias, una vez por semana, para que el lugar nunca se quede solo. Los grupos son heterogéneos; hay ejidatarios, artesanos, pescadores, pequeños propietarios, cañeros, trabajadores de restaurantes o del pequeño comercio y también de algunas empresas turísticas, sobre todo de

44 Entrevista a Teodoro -campesino de Jalcomulco- y José Lúcido, 29 de junio de 2015.

45 Entrevista a Lúcido, 26 de mayo de 2015, Campamento El Centinela, Carretera Coatepec-Jalcomulco, Veracruz, México.

46 Ricardo Delgado Salazar, "Los marcos de acción colectiva y sus implicaciones culturales en la construcción de ciudadanía”, Universitas humanística, 13, (julio-diciembre, 2007): http://revistas.javeriana.edu.co/ index.php/univhumanistica/article/viewFile/2165/1403 
aquellas de capital local, pues estas apoyan bastante el movimiento con la logística, recursos humanos y recursos materiales.

Cabe señalar que la organización interna se ha acuerpado a través de pequeños grupos que se diferencian por actividades, localidades, vecindades. Algunos grupos se reconocen con nombres como: Salvemos al rio, Patotas, Centinelas del río, Unidos vive el río, Bolis, Calle 20, Zaragoza, entre otros que conforman una red mayor en la que gran parte de sus miembros mantiene distintos niveles de participación.

La resistencia cuenta con diversos elementos de organización de actividades y roles, ya que para su composición se ha apoyado en la forma en que se planean y organizan las actividades en los campamentos turísticos, así como en la vinculación con otras organizaciones nacionales e internacionales como: el Tribunal Permanente de los Pueblos y el Movimiento Zapatista, y de activistas, personal académico y estudiantes de universidades. Por poner un ejemplo, las reuniones semanales provienen de la observación de las "Juntas del buen gobierno" con una relación horizontal que no permite un mando jerarquizado, sino la existencia de sujetos voceros en las asambleas rutinarias y representantes en las acciones de carácter legal. Es decir, practican un modelo de democracia directa y asamblearia con cargos rotativos y temporales y con una periodicidad frecuente de información a la totalidad de miembros sobre las actividades que se desarrollan, así se promueve la participación y la toma de decisiones. ${ }^{47}$

De las experiencias zapatistas incorporaron la idea de los caracoles, ya que los sonidos de "la caracola" ${ }^{48}$ les permiten mantenerse despiertos, alertas y en contacto con las demás comunidades por medio de la radio -walkie-talkie- o los teléfonos. Es decir, las caracolas simbolizan las formas de comunicación en los distintos medios para mantener la unión, solidaridad y comunicación entre simpatizantes en caso necesario.

Con el paso del tiempo, la distribución interna de los pequeños grupos hace mejor la resistencia y permite una acción colectiva más productiva, porque se optimizan más las tareas y hace eficaz la participación, debido a que se va consolidando la identidad de las redes de grupos que están en resistencia. Para mantener en términos de equidad el movimiento, cada uno de los grupos internos realiza guardias una vez por semana, el número de personas que se reúnen cada día son alrededor de 15 a 20 por horario diurno o nocturno; a lo que se agregan personas dedicadas a la cocina y visitantes.

47 Josué Lúcido, entrevistas con integrantes del campamento, 26 de mayo de 2015, Campamento El Tamarindo, Jalcomulco, Veracruz, México.

48 María Luisa Soriano González, "Organización y filosofía política de la Revolución Zapatista de Chiapas", Universitas. Revista de Filosofia, Derecho y Política (España) 18 (julio 2013): http://e-archivo.uc3m.es/ bitstream/handle/10016/17438/organizacion_soriano_UNIV_2013_18.pdf?sequence=1Cabe señalar que la artista Gypsipinta además de peces y caracoles que también simbolizan la comunicación. 
Mientras permanecen en la guardia, se rolan las actividades de boteo en la carretera, hacen leña, acarrean alimentos, alertan de la llegada de gente extraña, gente del gobierno o de la oposición al movimiento o de la presencia de individuos en el río, así como de la llegada de gente dispuesta a apoyar. Cabe señalar que los días lunes de cada semana tienen una reunión de información sobre los acontecimientos, donde realizan acuerdos que facilitan la organización, ya que todas las decisiones antes de ser tomadas se consensan en asamblea.

Otras formas de promover la información hacia la gente es el periódico mural que está ubicado en la calle principal de Jalcomulco y una bitácora dispuesta en el campamento, en esta bitácora se registra el monto del boteo, los ingresos y egresos diarios, el grupo participante de cada semana, y los eventos importantes del día.

Este orden y la planeación de sus roles de acción forman parte de las enseñanzas promovidas por las asociaciones y organizaciones señaladas anteriormente, sin olvidar que algunos sujetos de sus partícipes, como trabajadores de los campamentos en las empresas de turismo de aventura, han tenido un aprendizaje práctico de trabajo colaborativo, de organización y distribución para el aprovechamiento de los recursos: gente, tiempos y espacios, experiencias se han aplicado con éxito en el campamento.

El papel de las mujeres dentro de la resistencia es fundamental, puesto que dan cohesión al grupo, ya que su aportación no se reduce solamente a la preparación de los alimentos, limpieza de los espacios y apoyo a hijos y esposos, sino que también forman parte del comité de vigilancia diurna cuando llegan a hacer los alimentos y permanecen desde las 8 de la mañana a 5 de la tarde. Doña Margarita Garrido $^{49}$ dice que a ellas "no nos importa abandonar nuestros hogares mientras esté la lucha". Las mujeres que participan en el campamento están bien informadas y difunden las razones del porqué no quieren la presa, ya que les perjudicaría bastante en sus actividades, pues muchos viven de la venta de artesanías, comida y otros productos agrícolas. Por ello, doña Esther Soriano argumenta:

[...] que ojalá el gobernador nos escuche y pues... no seamos vendidos, porque prácticamente nos quiere vender a esa empresa, nosotros no estamos de acuerdo, porque los medios de comunicación, dicen que va a haber beneficios, pero esos beneficios, son para muy corto tiempo, [...] que nos dicen que nos van a dar un trabajo de muy corto tiempo, y nosotros no queremos trabajar, en esos empleos, porque nosotros tenemos fuentes de trabajo de donde vivimos. Nuestros esposos trabajan sus parcelitas en el río, sembramos semillas". ${ }^{50}$

49 Entrevista a Margarita Garrido, 26 demayo de 2014, Campamento El Tamarindo, Jalcomulco, Veracruz, México.

50 Entrevista a Esther Sánchez Soriano, de 7 de marzo de 2014, El Tamarindo, lugar del campamento, carretera Xalapa a Jalcomulco. 
El comentario de doña Esther expresa la preocupación generalizada por la situación y el conocimiento de que la construcción de la hidroeléctrica solo generará empleo precario y temporal, sin brindar provecho; al mismo tiempo que despojará de sus medios primarios de subsistencia a la población regional y su vida cotidiana se modificará.

Otra de las mujeres, doña Graciela, afirma que sus actividades están vinculadas tanto a la tierra como al río y expresa "que vivimos de la parcela donde se cultiva maíz, pipián, frijol, mango... todo lo que siembra se da" ${ }^{51}$ y menciona que cuando no hay trabajo "los hombres va a pescar camarón, se imagina si van a hacer todo esto [la presa], va a desviar el río y se va a disminuir o a acabar la pesca". ${ }^{52}$ Lo planteado por doña Graciela deja ver su preocupación sobre la posible escasez de los productos agrícolas básicos, pero sobre todo el riesgo de su soberanía alimentaria y de su relación con lo que produce el río que es elemental para la gastronomía de la localidad.

María, una de las mujeres que participa activamente en el campamento, comentó que lleva un diario personal en el cual describe las vivencias cotidianas que se suscitan en el campamento de El Tamarindo, donde ella señala cómo es alterada la forma de vida cotidiana de las personas en el día a día. Con esto se enfatiza que las mujeres, bien como madres y jefas de familia, o en su condición de mujeres, no solo tienen una participación como agentes activos del movimiento, sino que lo fortalecen brindándole unidad, sensibilidad y haciendo la estancia de los participantes menos dura en el campamento. A lo que se agrega que las mujeres, sin desatender sus labores domésticas, motivan a familiares, personas vecinas y a otras a interesarse y permanecer en el movimiento.

\section{Otras organizaciones participantes}

Para conocer el poder de convocatoria que tiene la resistencia, se realizó un simulacro en el campamento que sirvió para saber la cantidad de personas que lograrían reunir -en caso de ser necesario-, en el campamento de El Tamarindo. El simulacro tuvo una participación importante, pues se reunieron alrededor de 300 habitantes de Jalcomulco en su mayoría y unos cuantos, de otras poblaciones de la zona, en el plazo de pocos minutos.

En la participación activa del movimiento de resistencia destaca la población de Jalcomulco y de los poblados vecinos, en especial de habitantes de Teocelo con la Asociación Matlacobatl (AMA) de Teocelo, de igual manera a través de la Radio Teocelo se promueve el movimiento con la difusión y promoción de las actividades, anunciándolas en la radio.

51 Entrevista a Graciela, 8 de mayo de 2015, Campamento El Tamarindo, Jalcomulco, Veracruz, México.

52 Ibid. 
Aparte de la organización interna de Jalcomulco y la promoción de la radio señalada, también participan otras asociaciones, como ya se mencionó antes, para formalizar el movimiento la gente de otra comunidades se integró en organizaciones de grupos como Salvemos al río, los Centinelas del río y otros, enseguida se incorporó la organización no gubernamental de tipo ambientalista LAVIDA (Asamblea Veracruzana de Iniciativas y Defensa Ambiental) que se publicita como "un espacio plural de encuentro ciudadano, de análisis, denuncia y propuesta contra la devastación ambiental" 53 y en defensa de un ambiente sano. La organización está a cargo de Guillermo Rodríguez Curiel, ${ }^{54}$ quien mencionó que en la región también se conformó un Frente en Defensa de la Cuenca de La Antigua, en el que se incorporaron los comisariados ejidales, agentes municipales, comerciantes, alcaldes y uno que otro legislador, esta se conoce como Pueblos Unidos de la Cuenca de La Antigua por los Ríos Libres (PUCARL), organización que reúne a todos los demás pueblos, comunidades y municipios en jornadas informativas para hablarles acerca de la devastación que puede ocurrir pronto, si se permite la construcción de la presa cerca de Jalcomulco. ${ }^{55}$

En caso de que prosigan los estudios sobre el río con maquinaria de la empresa Odebrecht, los grupos mencionados, más las poblaciones vecinas acudirían en apoyo a la defensa del río, puesto que la información se brinda por distintos medios.

La agrupación LAVIDA implementó foros y talleres para brindar orientación del trabajo colaborativo y conocimiento sobre el desarrollo de las resistencias de otras regiones donde se plantea construir y mantener las presas. Lo importante es la defensa de la cuenca La Antigua, aunque cabe señalar que centinelas del río comentan que, en el caso de la orientación, fue solo una vez y que han estado al pendiente de la evolución del problema; pero solo como un apoyo externo, porque se han dado cuenta de que la organización que han establecido los distintos grupos de la red de PUCARL está bien planeada.

Así como La agrupación LAVIDA, el movimiento ecologista de Xalapa y Grupo Sendas Asociación Civil tienen por objetivo "promover y fomentar el desarrollo sustentable mediante el buen manejo de los recursos naturales, así como un desarrollo local con justicia y dignidad", ${ }^{56}$ al principio del movimiento implementaron foros de información, para apoyar el arranque de la resistencia de forma racional y colaborativa. Otro apoyo externo a la organización provino de personal académico de la Universidad Veracruzana y de estudiantes de la misma

53 "Qué es LAVIDA". Disponible en: http://www.lavida.org.mx/content/qu\%C3\%A9-es-lavida

54 Guillermo Rodríguez Curiel es un líder con conocimiento de las teorías y movimientos sociales, fue catedrático de la Facultad de Sociología en Universidad Veracruzana y también diputado por el Partido Revolución Democrática. Cabe señalar que en LAVIDA participan también personal académico de la Universidad Veracruzana y del CIESAS.

55 Elfego Riveros, “Activan plan contra presas en río Pescados-Antigua”, Altavoz, 17 de noviembre de 2013: http://periodicoaltavoz.blogspot.mx/2013/11/activan-plan-contra-presas-en-rio.html

56 Annie Demers Caron, "Eco-directorio de las Alternativas Ecológicas en Xalapa", Páginas Verdes Xalapa: http://www.paginasverdesxalapa.com/pdf/ecodirectorio.pdf 
institución, quienes, aparte de llevarles despensa para apoyar el movimiento, también les han brindado algunas ideas, formas de instrumentación y han permanecido en apoyo al movimiento. También se observa que hay organizaciones externas como MAPDER que es un Movimiento Mexicano de Afectados por las Presas y en Defensa de los ríos que desde 2004 surgió como organización de la sociedad civil vinculada con movimientos indígenas y campesinos que, con un modelo de desarrollo neoliberal sistemáticamente son objeto de diversos impactos sociales y ambientales en sus territorios. ${ }^{57}$ Esta asociación, con las experiencias y redes nacionales e internacionales como la REDLAR (Red Latinoamericana Contra Represas) y en defensa de los ríos y sus comunidades, a través de foros y encuentros ha logrado vincular a los movimientos de resistencia latinoamericanos para promover el fortalecimiento de los afectados por las presas y en defensa de los ríos. En estos foros Jalcomulco ha sido un centro importante de debates.

El movimiento de Jalcomulco ha sido afortunado en el sentido de que ha recibido apoyo económico y ha compartido las experiencias con otros movimientos. Estas organizaciones nacionales e internacionales promovieron foros con mucha audiencia, donde compartieron con movimientos estatales, nacionales e internacionales. Ya lo expresaba doña Bertha Anell, integrante de los centinelas del río, quien también dijo que el apoyo moral, social e incluso económico a la resistencia llega de varios lugares, México y Canadá principalmente, que envían despensas y colaboradores. ${ }^{58}$

Actualmente los problemas a los que se enfrentan las comunidades son la difusión de información parcial e inconclusa de parte de los organismos gubernamentales y del gobierno estatal, lo que genera un estado de confusión por parte de sus habitantes con menos información; la represión que ejerce el gobierno y la empresa Odebrecht a través del encarcelamiento de miembros del movimiento de defensa del rio, pues promueven la intimidación y difusión del miedo. De igual forma, las estrategias de desarticulación de la resistencia, utilizadas por la empresa en confabulación con el gobierno, han sido la infiltración de personas externas al grupo para crear conflictos. Tal es el caso de Telésforo Peña Marín, del Consejo de Vigilancia del Comisariado Ejidal de Jalcomulco, quien dice estar a favor de la construcción de la presa, por su relación con representantes de la empresa Odebrechtde: de esta manera, la empresa y el gobierno alientan la división, el enfrentamiento y la coerción para minimizar el movimiento.

Los grupos empresarios turísticos están en contra porque ven amenazados sus intereses y señalan de violencia toda acción de quienes defienden sus derechos, como a las poblaciones y a las organizaciones sociales, indígenas y

57 Gustavo Castro Soto “Nació MAPDER ¡El agua es nuestra, las represas la secuestran!”, EcoPor-tal.net, s.n. (2004): http://www.otrosmundoschiapas.org/analisis/ENMAPDERI.pdf

58 Entrevista a doña Bertha Anell, 26 de mayo de 2015, Campamento de El Tamarindo, Jalcomulco, Veracruz, México. 
campesinas que participan en el movimiento; incluso ven como favorable que el gobierno encarcele a líderes o representantes del movimiento con el afán de provocar miedo y coerción a sus participantes.

Parte de las estrategias empleadas por los entes interesados en la construcción de la hidroeléctrica es la desarticulación de la resistencia, a través de la confrontación de sus integrantes con la compra de terrenos cercanos al campamento.

Otra forma de resquebrajar la protesta es el convencimiento a habitantes de Tuzamapan de que el proyecto es confiable y beneficioso para la comunidad, porque les brinda un trabajo "seguro", así como la compra de terrenos en el predio donde se ubica el campamento e insisten a los ejidatarios en que lo mejor es que legalicen la propiedad de sus parcelas y vendan sus terrenos para aprovechar el capital, para lo cual deben acudir a las oficinas del PROCEDE, el cual, a través de los cambios del artículo 27 constitucional sobre la propiedad privada, puede establecer sus terrenos como propios; pero eso no significa que pierdan la relación y participación en el comisariado ejidal, ni que dejen de hacer su trabajo comunitario.

Los entes empresarios envían a su personal a colocar trípticos en lugares estratégicos con el fin de promover "las bondades" o los beneficios del proyecto hidroeléctrico; como dice el presidente de Jalcomulco: "colocan trípticos de sí a las presas, como si fuera una campaña política" ${ }^{59}$ Es más, el presidente municipal ha sido criticado por el sector empresarial por su activismo, incluso fue sancionado por autoridades superiores por su participación en el movimiento. Por distintos medios se ha buscado la desunión de la autoridad municipal con el movimiento, creando un ambiente hostil entre este y habitantes; sin embargo, él, como un habitante más de Jalcomulco, siente afectado su territorio, economía y vida cotidiana, de ahí que los apoya en la lucha como lo comprueba el hecho de participar en el campamento con su familia, ya que su esposa, como otra mujer más, colabora en la preparación de los alimentos.

A pesar de todo ello, la organización ha respondido manteniéndose firme ante estas presiones y coerciones informándose y asesorándose jurídicamente con abogados ambientalistas y penalistas, para que no los hagan caer en falacias que rompan la armonía del movimiento, pero también para buscar los elementos necesarios que les den garantías de que la empresa Odebretch abandone este proyecto, como indicó José Luis Hernández, quien por ahora es uno de los representantes del movimiento. ${ }^{60}$

Y como dice Josué Lucido, “ya sabemos que el impacto ambiental, social y económico son [sic] mayores al beneficio por este proyecto y por eso la gente permanece en el plantón y seguirán durante el tiempo que sea necesario e incluso defenderán con su vida si es preciso". ${ }^{61}$ Por el lado del gobierno sigue habiendo

59 Entrevista a Josué Lúcido, 8 de mayo de 2015, Campamento El Tamarindo, Jalcomulco, Veracruz, México.

60 Entrevista a José Luis Hernández, 29 de junio de 2015, Campamento El Tamarindo, Jalcomulco, Veracruz, México.

61 Ibid. 
una ausencia de información; mientras que la prensa señala que el dueño de la empresa Odebretch está preso en Brasil por actos de corrupción; pero mientras no haya claridad en detener la construcción de la presa, la gente se mantiene firme en continuar con el campamento y, como afirman, los roles están calendarizados, lo mismo que las asambleas, al menos, para todo el año 2016.

\section{Conclusiones}

La acción colectiva es la base de los movimientos sociales; esta es el principal recurso y, con frecuencia, el único del que dispone la mayoría de la gente para enfrentarse a su adversario. Las formas de acción colectiva son tan heterogéneas como las formas de represión y control social empleados para combatirla, y en el caso de Jalcomulco se cumple esta premisa.

En la medida en que la empresa no logre sus propósitos, la resistencia de Jalcomulco tiene éxito y este éxito radica en la pluralidad de los actores participantes, puesto que la lucha de las poblaciones afectadas, apoyadas por el presidente municipal, ha estado reforzada con la participación de diversas organizaciones no gubernamentales como LAVIDA, activistas de SENDAS, personal investigador de la Universidad Veracruzana, y del Centro de Investigaciones y Estudios Superiores en Antropología Social (CIESAS), además de estudiantes con compromiso social, sin olvidar la contribución internacional de la REDLAR y la nacional de MAPDER. Esto le ha conferido al movimiento una articulación particular de apoyo a la resistencia, a lo que se agrega su proyección ante medios de comunicación tanto al interior de la República como hacia afuera.

La reflexión por parte de los sujetos integrantes más activos del movimiento ha favorecido su rumbo, puesto que las distintas acciones que se han llevado a cabo se han difundido en los municipios afectados con la intensión de que la gente tome conciencia de los impactos ambientales, económicos y sociales por la construcción de la presa, pues en general la población carecía del conocimiento suficiente de las afectaciones que un proyecto de tal medida puede tener en un territorio.

La existencia de un estado de ánimo equilibrado del movimiento socioambiental hace referencia a la disposición que tiene la población de un territorio de apoyar determinado tipo de protesta, de acuerdo con las circunstancias sociales y económicas que existan. En el caso de la protesta analizada, se observa que el movimiento ha tenido una óptima receptividad, producto del crecimiento económico obtenido con el turismo de aventura y las otras actividades productivas de la zona de Jalcomulco; pero también por el ánimo de las personas que, por su apego, defienden el río, pues ahí han vivido generaciones tras generaciones. 